\title{
Recurrent Nasopharyngeal Undifferentiated Carcinoma
}

National Cancer Institute

\section{Source}

National Cancer Institute. Recurrent Nasopharyngeal Undifferentiated Carcinoma. NCI Thesaurus. Code C8231.

The reemergence of undifferentiated carcinoma of the nasopharynx after a period of remission. 